\title{
Final state hadronic rescattering with UrQMD
}

\author{
J. Steinheimer ${ }^{1, \star}$, V. Vovchenko ${ }^{1,2}, J$. Aichelin ${ }^{1,3}$, M. Bleicher ${ }^{1,2,5}$, and H. Stöcker ${ }^{1,2,4}$ \\ ${ }^{1}$ Frankfurt Institute for Advanced Studies, Ruth-Moufang-Str. 1, 60438 Frankfurt am Main, Germany \\ ${ }^{2}$ Institut für Theoretische Physik, Goethe Universität Frankfurt, Max-von-Laue-Strasse 1, D-60438 Frankfurt am Main, Germany \\ ${ }^{3}$ SUBATECH, UMR 6457, Université de Nantes, Ecole des Mines de Nantes, IN2P3/CNRS. 4 rue Alfred Kastler, 44307 Nantes \\ cedex 3, France \\ ${ }^{4}$ GSI Helmholtzzentrum für Schwerionenforschung GmbH, D-64291 Darmstadt, Germany \\ ${ }^{5}$ John von Neumann Institut für Computing, FZ Jülich, 52425 Jülich, Germany
}

\begin{abstract}
In this talk we discuss the effects of the hadronic rescattering on final state observables in high energy nuclear collisions. We do so by employing the UrQMD transport model for a realistic description of the hadronic decoupling process. The rescattering of hadrons modifies every hadronic bulk observable. For example apparent multiplicity of resonances is suppressed as compared to a chemical equilibrium freeze-out model. Stable and unstable particles change their momentum distribution by more than $30 \%$ through rescattering. The hadronic rescattering also leads to a substantial decorrelation of the conserved charge distributions. These findings show that it is all but trivial to conclude from the final state observables on the properties of the system at an earlier time where it may have been in or close to local equilibrium.
\end{abstract}

\section{Introduction}

The aim of ultrarelativistic heavy ion experiments at the Relativistic Heavy Ion Collider (RHIC) and the Large Hadron Collider (LHC) is is the study of the properties of the socalled quark gluon plasma (QGP) which is created for a short time in these reactions [1-5]. This includes the determination of the yet unknown transport properties of QCD matter as well as the study of its phase structure at large net baryon densities. It involves the study of hadron multiplicities but also fluctuations of conserved quantities in a given rapidity interval which have been compared with $1 Q C D$ results

In the theoretical description of these reactions we are confronted with rapidly expanding systems which may or may not hadronize at (or near) the crossover line (chemical freeze out) and continue to expand until the interactions cease. This latter phase is called hadronic phase and is characterized by hadronic interactions which govern the time period when the system drops out of equilibrium. To draw meaningfull conclusions one has to assess how the hadronic phase has modified the observables.

To do so sophisticated model simulations are usually employed. The current state-of-the-art of such models are so called hybrid models where a (viscous) fluid dynamical simulation [6-9] is followed by an expanding gas of hadrons. The evolution of such a hadron gas can be

${ }^{\star}$ e-mail: steinheimer@fias.uni-frankfurt.de described with the relativistic Boltzmann equation:

$$
p^{\mu} \cdot \partial_{\mu} f_{i}\left(x^{\nu}, p^{\nu}\right)=C_{i}
$$

Here the change of the single particle distributions $f_{i}\left(x^{v}, p^{v}\right)$ (left hand side) is caused by the collision term $C_{i}$ (right hand side). Using a transport model (based on the Boltzman equation) as an afterburner which follows the fluid dynamical evolution has become the standard method of treating the interactions during the hadronic phase of nuclear collisions [6, 8-15]. Recently several studies have pointed out the importance of the hadronic rescattering, during the freeze-out phase of a nuclear reaction, on the description of observables like particle numbers and spectra, resonance production, momentum anisotropies and particle number fluctuations [16-19].

This talk will give a summary of how the hadronic interactions affect the final state observables. The results presented can be found in $[20,21]$ where a more detailed description and discussion is presented.

\section{UrQMD}

To study the dynamics of the hadronic phase we will use the UrQMD hybrid model [9]. This model combines a $3+1 \mathrm{D}$ ideal fluid dynamical simulation with a state-of-theart transport description of the non-equilibrium hadronic decoupling phase. In the present investigation we use the 


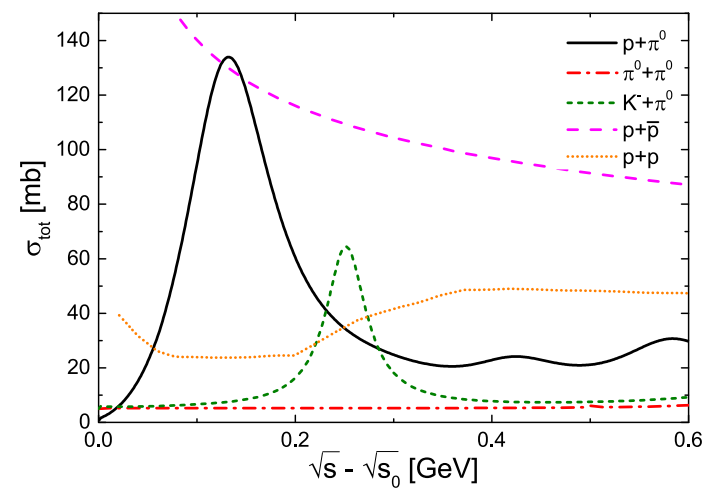

Figure 1. Different total (elastic + inelastic) hadronic cross sections as implemented in UrQMD. We show the cross sections as function of the relative energy, i.e. we subtract the rest masses $\sqrt{s_{0}}$ of both hadrons from the total invariant mass.

Cooper-Frye equation,

$$
E \frac{d N}{d^{3} p}=\int_{\sigma} f(x, p) p^{\mu} d \sigma_{\mu}
$$

to transform the fluid dynamical fields to discrete hadrons on an iso-energy density hypersurface [22] $e_{C F} \approx 350$ $\mathrm{MeV} / \mathrm{fm}^{3}$. Here $f(x, p)$ corresponds to the grand canonical Bose- or Fermi- distribution functions, depending essentially on the local temperature $T(x)$ and chemical potentials $\mu(x)$. To obtain a finite number of particles the integral is randomly sampled, taking into account the global cosnervation of baryon number strangeness and electric charge.

The non-equilibrium transport part of the UrQMD model is based on $2 \rightarrow n$ hadronic scattering, according to measured reaction cross sections [23], which serve as the main input to the model. The model includes nearly 60 different baryonic species with their anti-particles as well as about 40 mesonic states [24, 25]. The possible interactions between these hadrons include elastic scattering, resonance excitations, string excitations as well as strangeness exchange reactions. To present an example of the cross sections implemented in the UrQMD model we show the total cross sections for several processes in Fig. 1. As one can see there are several processes with very large cross sections, mainly involving protons. In particular the proton+anti-proton annihilation cross section is large. The probabilities of certain reactions do not only depend on the magnitude of the cross section but also on the phase space densities of the involved hadrons, making the microscopic description of the hadronic phase so important.

\section{Results}

In the following the dynamics of the hadronic interactions in the final phase of collisions of $\mathrm{Au}+\mathrm{Au}$ at beam energies of $\sqrt{s_{N N}}=200 \mathrm{GeV}$ are investigated, focusing on the mid

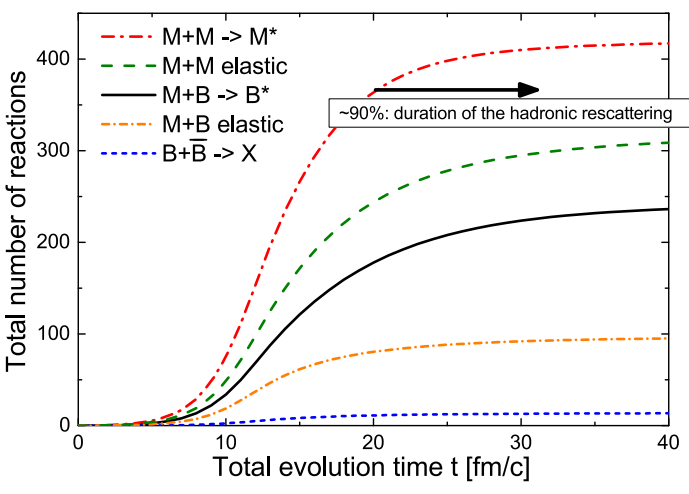

Figure 2. Total number of different hadronic interactions occurring until the time $t$, during the hadronic phase of central collisions of $\mathrm{Au}+\mathrm{Au}$ at $\sqrt{s_{N N}}=200 \mathrm{GeV}$.

rapidity region $-0.5<y<0.5$ of most central $(b<3.4$ $\mathrm{fm})$ collisions where experimental data are available.

The total number of hadronic scatterings, up to a time $t$, defined as the time since the first initial collisions have taken place, is shown in figure 2. Here different possible hadronic interactions, meson+meson resonance excitation and elastic scattering, meson+baryon resonance excitation and elastic scattering as well as baryon+antibaryon annihilation are compared. The final rescattering is dominated by meson+meson interactions and only very few annihilation processes take place. The number of scatterings changes most drastically in the time between hadronization, around $10 \mathrm{fm} / \mathrm{c}$, and a time of roughly $20 \mathrm{fm} / \mathrm{c}$, after which the number of hadronic scatterings does essentially not change anymore. This means that the hadronic rescattering mainly takes place in the $10 \mathrm{fm} / \mathrm{c}$ after hadronization.

\subsection{Resonance dynamics}

If one wants to experimentally verify the existence and duration of the hadronic phase, the study of the production and absorption rates of hadronic resonances in nuclear collisions is a useful tool [26]. Resonances which decay early during the hadronic phase may not be identified experimentally because the decay products rescatter. Therefore the hadronic intercations are reflected in the number of identified resonances. The states discussed in this work, are summarized in table 1 .

It is obvious that the listed hadronic resonances have widely varying properties and in particular different lifetimes which should be reflected in the dynamics of these resonances during the hadronic phase. In figure 3, the net resonance gain rate as a function of time for the different hadronic resonances is shown. The net gain rate is defined as

$$
\frac{1}{N_{\mathrm{CF}}}\left(\frac{d N_{\text {gain }}}{d t}-\frac{d N_{\text {loss }}}{d t}\right)
$$




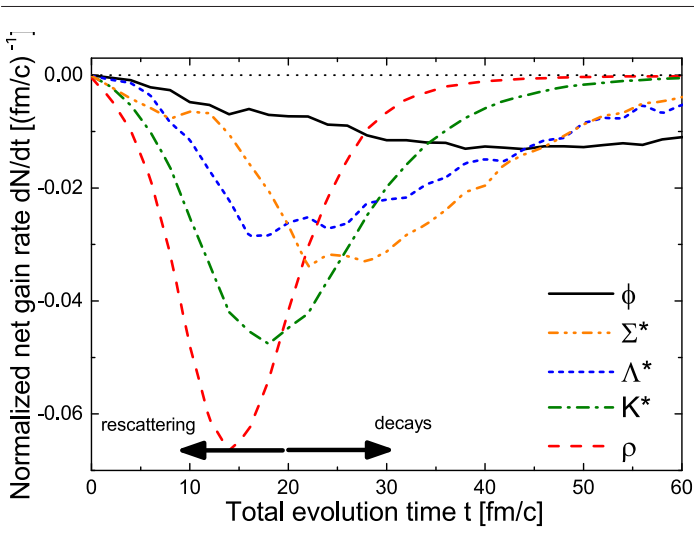

Figure 3. Net gain rates of different hadronic resonances as function of time and normalized to the initial yield at hadronization (Cooper-Frye). The results are for central collisions of $\mathrm{Au}+\mathrm{Au}$ at $\sqrt{s_{N N}}=200 \mathrm{GeV}$.

where $N_{C F}$ is the resonance yield at hadronization (Cooper-Frye), $\frac{d N_{\text {gain }}}{d t}$ is the number of reactions that create a resonance and $\frac{d N_{\text {loss }}}{d t}$ is the number of reactions that destroy a resonance, e.g. decay or inelastic scattering, per unit of time. The results presented in figure 3 clearly reflect the different lifetimes of the resonances, as the $\rho$ meson shows its largest loss rate during early times, while the $\phi$ decays only slowly and therefore shows a slow loss. However there is an interesting comparison between the $\Lambda^{*}$ and $\Sigma^{*}$. Even though there is a lifetime difference of a factor 2 between these states, their net gain rate appears to be very similar. This hints to the fact that the $\Sigma^{*}$ has a quite substantial regeneration rate at early times of the hadronic phase.

The lifetime hierarchy can also be observed in figure 4 where we show the fraction of observable/reconstructable resonances which have decayed until a time $t_{D}$, as function of time. As expected, the $\phi$ meson decays at a very late time and the short lived resonances decay at an earlier time. The difference of the $\Sigma^{*}$ and $\Lambda^{*}$ is smaller than expected due to the significant $\Sigma^{*}$ regeneration at early times. An important observation is that even though short lives resonances like the $\rho$ or $K^{*}$ are the first to decay, most of the observable resonances still decay at times $t>15 \mathrm{fm} / \mathrm{c}$, i.e. a rather dilute hadronic system and hence may not

Table 1. Resonances and their decay channels

\begin{tabular}{|l|c|c|c|}
\hline Resonance & decay & $\begin{array}{c}\text { branching } \\
\text { ratio }\end{array}$ & $\begin{array}{c}\text { lifetime } \\
{[\mathrm{fm} / c]}\end{array}$ \\
\hline$\rho(770)^{0}$ & $\pi^{+}+\pi^{-}$ & 1 & 1.335 \\
$K^{*}(892)^{0}$ & $\pi^{-}+K^{+}$ & 0.67 & 4.16 \\
$\phi(1020)$ & $K^{+}+K^{-}$ & 0.489 & 46.26 \\
$\Sigma(1385)^{+}$ & $\pi^{+}+\Lambda$ & 0.870 & 5.48 \\
$\Sigma(1385)^{-}$ & $\pi^{-}+\Lambda$ & 0.870 & 5.01 \\
$\Lambda(1520)$ & $K^{-}+p$ & 0.225 & 12.54 \\
$\Xi(1530)^{0}$ & $\pi^{+}+\Xi^{-}$ & 0.67 & 22 \\
\hline
\end{tabular}

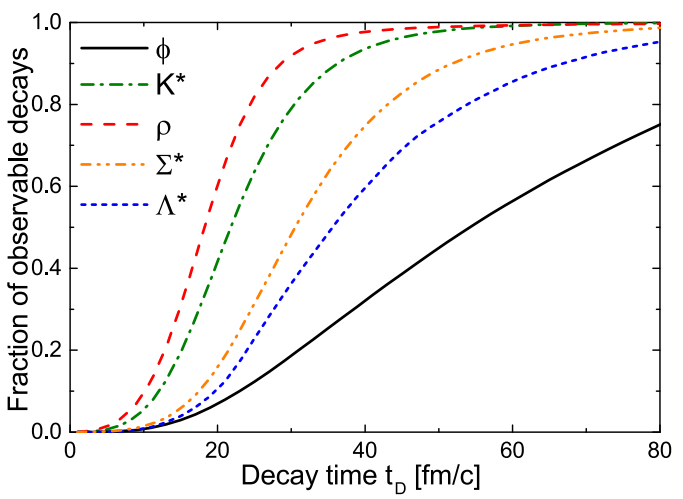

Figure 4. Fraction of observable resonances which have decayed until a time t. The results are for central collisions of $\mathrm{Au}+\mathrm{Au}$ at $\sqrt{s_{N N}}=200 \mathrm{GeV}$.

carry much information on the dense system where they where born.

Not only do these resonances have different lifetimes, but they also decay into different daughter particles which have to be identified in order to reconstruct the original resonance. As these daughter particles also undergo rescattering they may be lost to reconstruction, thus also the information on the mother resonance would be lost. The probability of the daughter particle rescattering does depend on the time of the resonance decay and on the rescattering cross section of the daughter particles which is different for example for pions and kaons.

Not all resonances decay at the same time and therefore the detection probability for the different resonances will depend on their lifetime. In figure 5 the time integrated detection probability is shown as a function of the transverse momentum $p_{T}$ of the resonance. There is a clear hierarchy of the detection probability of the resonances where the $\phi$ with its long lifetime and small hadronic cross section has the highest and the $\rho$ has the lowest probability of being detectable.

Will the final state interactions lead to observable differences in resonance multiplicities and properties? To answer this question we study the ratio of the $p_{T}$ integrated multiplicity of detectable resonances and the corresponding multiplicity of their ground state hadron in figures 6 and 7 . Here the results are compared with experimental data from the STAR experiment [27-29]. A very good agreement of our results with the experimental data in the case of the full model is observed. When the final hadronic rescattering is neglected there is a deviation from the experimental results, especially for the $K^{*}$ and $\Lambda^{*}$ ratios, indicating that they are most sensitive to the rescattering phase.

\subsection{Stable hadrons}

In the following we will discuss whether we can also expect an impact of the rescattering on stable hadron yields 


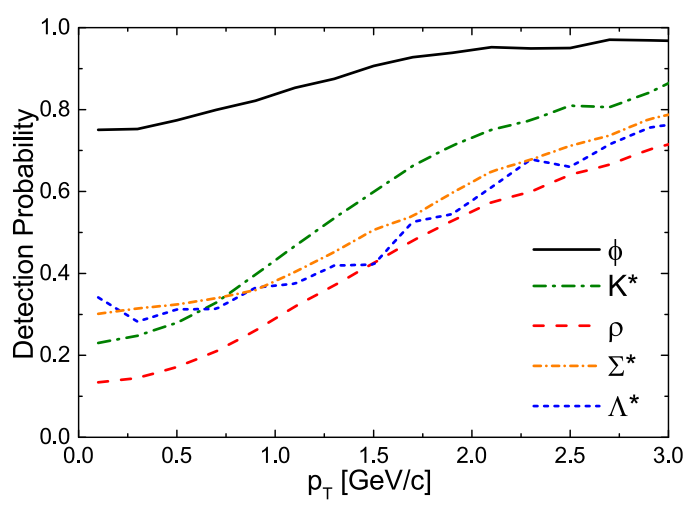

Figure 5. Detection probability of different hadronic resonances as function of their transverse momentum. A resonance is detectable if none of their decay products rescatter in the hadronic phase. The results are for central collisions of $\mathrm{Au}+\mathrm{Au}$ at $\sqrt{s_{N N}}=$ $200 \mathrm{GeV}$.

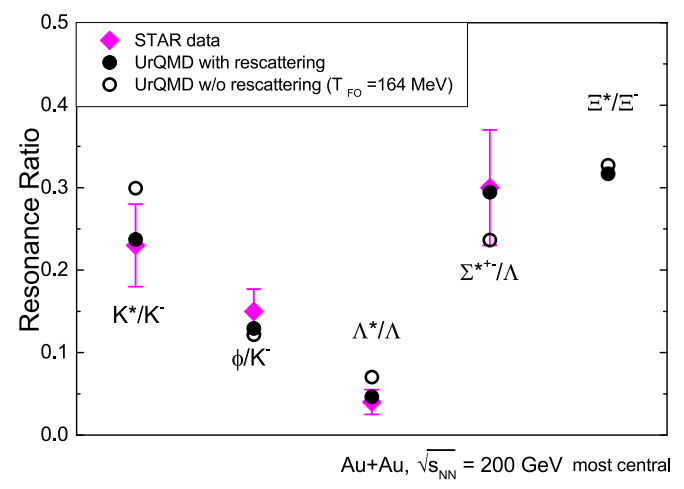

Figure 6. Ratios of detectable resonances to their ground state after the hadronic phase (full circles) and before hadronic rescattering (open circles), compared to data from the STAR experiment (magenta diamonds).

and properties like spectra and collective flow. Here one can distinguish two types of hadronic scatterings. One is the (pseudo-)elastic scattering which changes only the momentum distribution of the present hadrons but not their abundances. There is also be inelastic scatterings which change the flavor and hadronic abundances during the hadronic rescattering. The most relevant processes her is the annihilation of baryons and anti-baryons. But also strangeness exchange reactions and multi-body decays of resonances increasing the number of pions. Figure 8 we show the time dependence of stable hadron yields (including contributions from resonance decays) as a function of time, for most central collisions of $\mathrm{Au}+\mathrm{Au}$ at a beam energy of $\sqrt{s_{N N}}=200 \mathrm{GeV}$. The multiplicities are scaled to the final (observable) value to quantify the effect of the hadronic rescattering and the clear time dependence confirms that the hadronic rescattering changes the chem-

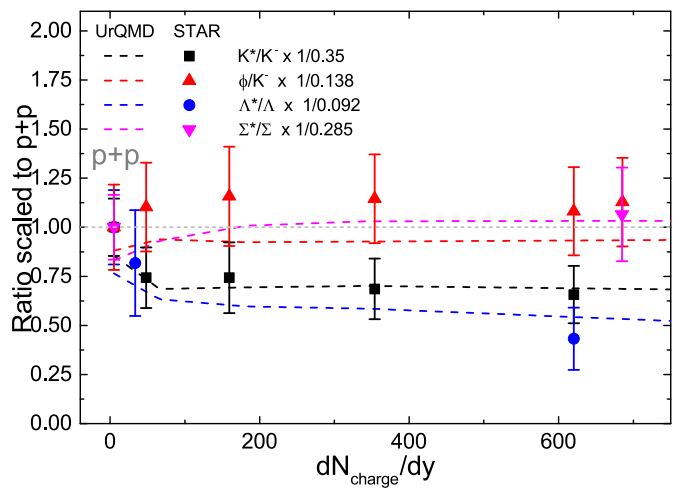

Figure 7. Ratios of detectable resonances to their ground state after the hadronic phase as function of centrality (lines) compared to experimental data (symbols). The results are for collisions of $\mathrm{Au}+\mathrm{Au}$ at $\sqrt{s_{N N}}=200 \mathrm{GeV}$. The quoted temperature $T_{F O}$ corresponds to the $\epsilon \approx 350 \mathrm{MeV} / \mathrm{fm}^{3}$ criterion.

ical composition of the stable hadrons to times as large as $20 \mathrm{fm} / \mathrm{c}$. For example the protons show the largest decrease in yield due to annihilation, even though the total baryon number is always exactly conserved. The proton annihilation is stronger than that of $\Lambda$ hyperons due to the larger annihilation cross section. In fact a clear hierarchy due to the decrease of the cross section with increasing strangeness is visible.

The effect of hadronic rescattering on the final observed momentum spectra is shown in figure 9. Here the change of the spectra is shown as the ratio of the final $p_{T}$ spectrum over the spectrum at chemical freeze out (Cooper-Frye). We compare three distinct hadrons, pions, protons and $\Omega$. Each species shows a very different characteristic change of the spectrum. The proton spectrum is mainly enhanced at large momenta and is depleted at low momenta, which reflects the generation of transverse collective velocity flow which has been observed in these reactions. This change is mainly caused by elastic and pseudo-elastic scatterings, while baryon antibaryon annihilation decreases the spectrum mostly independent of $p_{T}$. The pions also obtain an additional radial flow, however also the low $p_{T}$ part of the pion spectrum is enhanced. This is due to contributions from resonance decays which populate mainly the low transverse momenta. The $\Omega$ baryon receives the smallest change in the spectrum due to its small hadronic rescattering cross section. It is little affected from the transverse flow. That the average value of the ratio is below one is likely a consequence of the strangeness exchange reaction and longitudinal expansion.

\subsection{Annihilation}

An important and much discussed contribution to the final state hadronic rescattering is the baryon+anti-baryon annihilation reaction. In this reaction the baryons, e.g. proton and anti-proton, annihilate to form multiple mesons. 


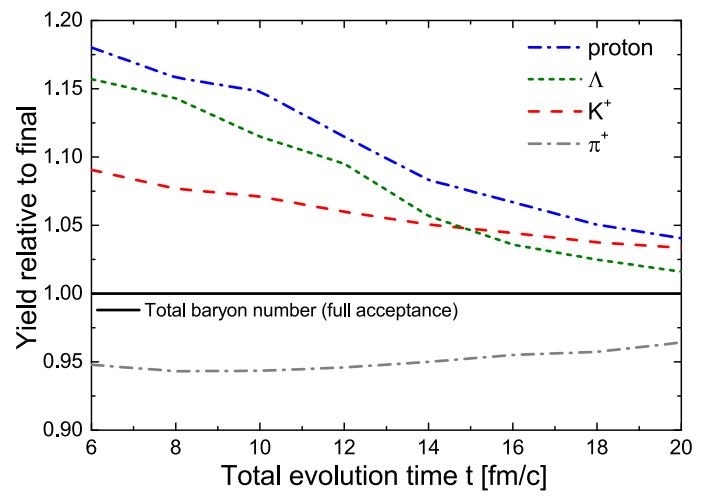

Figure 8. Time dependence of the stable hadron yields (including their resonance feed down), normalized to the final yields, during the hadronic phase. The results are for central collisions of $\mathrm{Au}+\mathrm{Au}$ at $\sqrt{s_{N N}}=200 \mathrm{GeV}$.

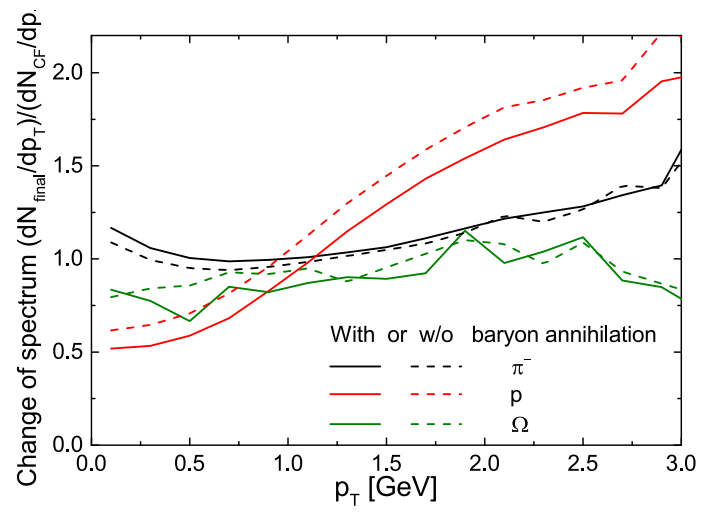

Figure 9. Change of the spectrum of stable hadrons due to the finals state rescattering. We compare two scenarios, one including the baryon+antibaryon annihilation and one excluding the annihilation reaction. The results are for central collisions of $\mathrm{Au}+\mathrm{Au}$ at $\sqrt{s_{N N}}=200 \mathrm{GeV}$.

For the case of nucleons and anti-nucleon on average about 5 pions are created. Since most transport models (as UrQMD) do not include multi particle scatterings, the inverse reaction, 5 pions creating a baryon + antibaryon pair, is not taken into account [30-33]. This violates detailed balance but in an expanding system which hadronizes at an energy density of $350 \mathrm{MeV} / \mathrm{fm}^{3}$ this reaction, which decreases with $\rho^{5}$, is rare [34]. This has also been shown in studies where the detailed balance for this reaction has been explicitly included and the resulting baryon and anti-baryon loss in the hadronic phase is comparable to our scenario [35]. Nevertheless it is worthwhile to further pursue the implementation of a hadronic rescattering phase that includes such multi-particle scattering in order to minimize the systematic errors on the model side.

\section{Fractions of annihilated baryons}

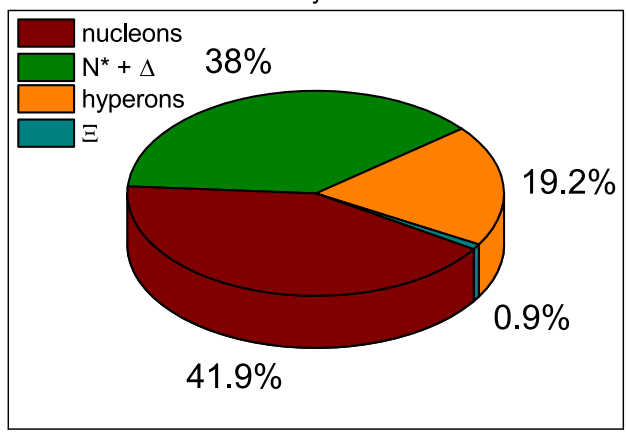

Figure 10. Relative fraction of different baryonic species lost due to baryon+antibaryon annihilation in the hadronic phase. Only $42 \%$ of all annihilated baryons are actual nucleons. The rest is composed of excited nucleonic states as well as hyperons and their resonance states. As a result less than 25 percent of all annihilations actually takes place between two nucleons in their ground state. The results are for central collisions of $\mathrm{Au}+\mathrm{Au}$ at $\sqrt{s_{N N}}=200 \mathrm{GeV}$.

For simplicity one usually argues about the annihilation of ground state nucleons, and possibly hyperons, but generally one neglects that a significant portion of baryons consists of excited resonance states with significant larger masses. For example at LHC and RHIC at chemical freeze out almost half of all baryon exist in form a baryonic resonance. In our approach the absolute value and the $\sqrt{s}$ dependence of the annihilation cross section for the resonances is assumed to be identical to that of the ground state with the same quantum numbers. Figure 10 depicts the percentage of the different annihilation channels which contribute to the annihilation. One can clearly see that less than half of all annihilated baryons are actually in their ground state. This also means that in less than $25 \%$ of all annihilation reactions two ground state baryons annihilate. The majority of these reactions will have a larger invariant mass and therefore produce on average more than 5 pions and eventually even heavier mesons like kaons. Thus the back reaction of these annihilation reactions will be even more suppressed by the phase space density and also the effective implementation of all possible processes is much more involved.

\subsection{Effects on fluctuations of conserved charges}

As a last point we will discuss the effects on the distribution of conserved charges. We set up a time-dependent correlation function to explore the diffusion of conserved charges during the hadronic phase. Following the standard definition of the correlation coefficient this function reads as

$$
r_{\mathrm{IF}}(t)=\frac{\sum_{n}\left(I_{n}(t)-\bar{I}(t)\right)\left(F_{n}-\bar{F}\right)}{\sqrt{\sum_{n}\left(I_{n}(t)-\bar{I}(t)\right)^{2} \sum_{n}\left(F_{n}-\bar{F}\right)^{2}}},
$$




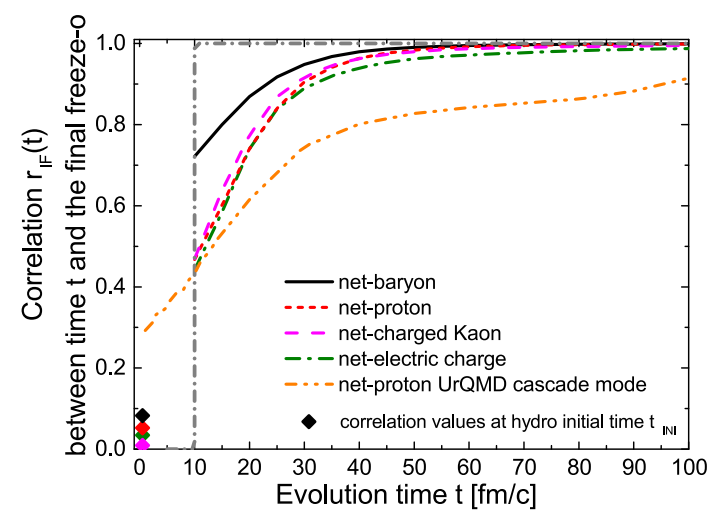

Figure 11. Correlation of the net-charge, net-kaon, net-proton and net-baryon number in the rapidity interval $-0.5<y<0.5$ with an earlier time $t$ in that same rapidity interval.

where $I_{n}(t)$ denotes the number of a given charge (or a netparticle number) in a given rapidity and momentum window at the time $t$ in the event number $n . F_{n}$ is the final number of that charge after all interactions have ceased, in the same event. Finally, the $\bar{I}(t)$ and $\bar{F}$ are the corresponding averages. The sum runs over all events in the sample.

It is evident that if the net-charge in a given bin at a given time $t$ is perfectly correlated with the net-charge at the end of the evolution, then the value of this correlation function $r_{\mathrm{IF}}(t)$ will be equal to 1 whereas if the net-charge number at given time $t$ is completely uncorrelated with the final value, then the $r_{\mathrm{IF}}(t)$ will be equal to zero.

In the standard scenario [36], which is employed in most comparisons of the (normalized) cumulants with the HRG or lattice QCD results one essentially assumes that this correlation is equal to zero before the chemical freezeout of hadrons and then instantly jumps to one at the chemical freeze-out, a value which does not change until the cumulants are observed by experiment. This scenario is shown in figure 11 by a gray dash dotted line for an exemplary freeze-out time of $10 \mathrm{fm} / \mathrm{c}$. In such a scenario the final value of the cumulants correspond to that at chemical freeze-out, without any information about the QGP phase before freeze-out or about the hadronic rescattering which follows hadronization.

To estimate the effect of the full local equilibration on the decorrelation of the different charges we will also contrast the hybrid model simulations with results obtained from the standard UrQMD model in its cascade version. In the cascade version of the model, the system remains essentially out of local equilibrium for a long time and, due to the finite cross sections, will retain more information and therefore show a stronger correlation with the early times.

Figure 11 shows the dependence of the correlation $r_{\mathrm{IF}}(t)$, for different net-charge and net-particle numbers, as a function of time. Unlike in the standard scenario assuming instant freeze-out, the correlation function strongly depends on the duration of the rescattering phase for all con-

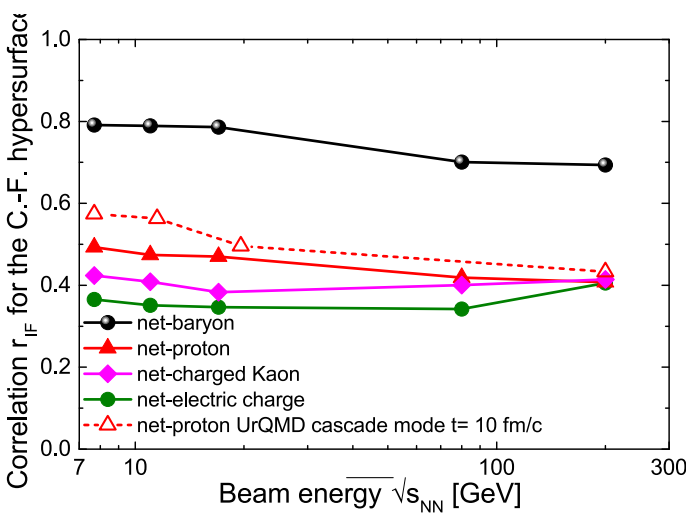

Figure 12. Correlation of the finally observed net-charge, netkaon, net-proton and net-baryon number in the rapidity interval $-0.5<y<0.5$, at the Cooper-Frye hypersurface, with the final net-charge density as a function of beam energy.

sidered quantities. At very late times $(t>40 \mathrm{fm} / \mathrm{c})$ the correlation function approaches 1 , which is expected as the system has essentially frozen out and interactions as well as resonance decays have ceased by that time. However, the correlation changes rapidly in the time interval between particle production (on the Cooper-Frye hypersurface) at around $10 \mathrm{fm} / \mathrm{c}$ and decoupling of the system at about $25 \mathrm{fm} / \mathrm{c}$. At the time of interest for most studies, namely the particle production time, the correlation is approximately 0.5 for most net-charges. Only the net-baryon number correlation is slightly stronger. However, the quantity that is measured experimentally, namely the net-proton number, shows a similar correlation as the net-charge, as significant iso-spin changing reactions take place during the rescattering phase. One should keep in mind that the fact that the correlation still changes at rather late times does not mean that the lifetime of the hadronic phase is on the order of $40 \mathrm{fm} / \mathrm{c}$. At a time of $20 \mathrm{fm} / \mathrm{c}$ about $90 \%$ of all 2-body reactions have already occurred. After that we observe mainly resonance decays.

There is a crucial point in time at which it is worthwhile to study the correlation $r_{\mathrm{IF}}(t)$ in more detail. It is the time at which hadrons are produced according to chemical equilibrium distributions. At this point, at the transition between the hadronic and quark phase, the hadrons are sampled from the transition (hadronization) hypersurface according to the Cooper-Frye equation. In fact since the iso-energy hypesurface does not correspond to a fixed time we would not compare the net charge at two different fixed times, but on the hypersurface volume and in the final state. The second one is an even earlier time at which the fluid dynamical evolution starts. Therefore we show in figure 12 the correlation $r_{\mathrm{IF}}\left(t=t_{\mathrm{CF}}\right)$ which is defined as the correlation between the net-particle number going through the Cooper-Frye hypersurface and the final state particle number. Again this correlation does not compare two fixed times but two 4-volumes in which the relevant quantities are conserved globally. The correlation 


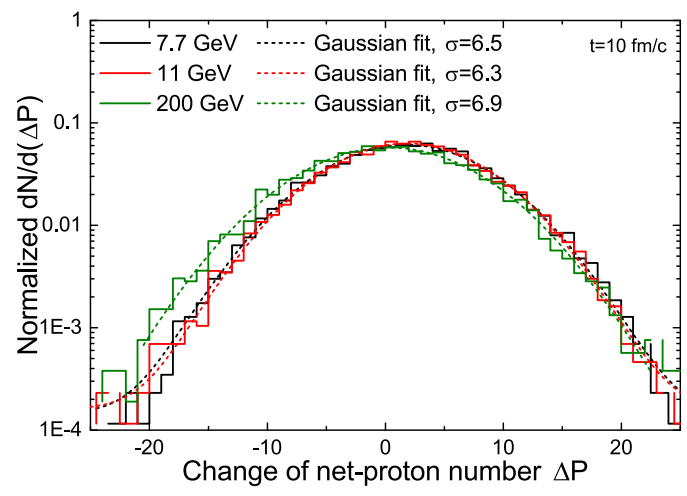

Figure 13. Distribution of the change of the net proton number in a given event, for different beam energies.

between the Cooper-Frye surface and the final state apparently shows only a very weak beam energy dependence down to $\sqrt{s_{\mathrm{NN}}}=7 \mathrm{GeV}$. This means that the importance of the hadronic rescattering does not change much within the beam energy range of the beam energy scan program at RHIC.

As a last step we will demonstrate the effect of the de-correlation of the different net-charge and net-particle numbers on their final number distributions. To do so we show the distribution of the change of a particular netparticle number (i.e. the net-proton number) for different beam energies. The change of the net-proton number $\Delta P$ is defined as the final net-proton number in a single event minus the net-proton number at a given time $t$ in that single event. Figure 13 shows that this distribution function for the change of the net-proton number has a broad distribution. We were able to fit this distribution with a Gaussian of width $\sigma=6.5$, which is only slightly smaller than the width of the final net-proton number distribution which is 7.5. The width of the Gaussian smearing is approximately constant for all beam energies, which is consistent with the observation in figure 12 that the de-correlation is almost independent of beam energy.

\subsection{Relevance for observations}

At this point we want to put our results in context with previous publications $[37,38]$ on interpreting data from heavy ion collisions. It is important to keep in mind that, even though we have shown that the actual net-charge number in an acceptance window will change in every event due to the rescattering, this does not necessarily mean that also the measured cumulant of that net-charge will also change. Let us take for example a system which does not entail any correlations between particles. Then the cumulants of any order are merely the mean number of that charge (see e.g. [39]). So if the mean particle number is fixed at some point during the evolution the cumulants for a non correlated system will be fixed by definition at that same point, called the chemical freeze out point (not to be mistaken with the latest point of chemical equilibrium).
If the system has strong correlations present at the point where the mean particle numbers are fixed, due to a phase transition or critical endpoint, and these correlations are washed out by the hadronic rescattering, again the cumulants will simply reflect the mean values. Note that there is a small contribution to the cumulants from conservation laws. These anti-correlation effects are small for high beam energies and they essentially cancel when taking ratios of same order cumulants, thus they are not relevant for the discussion presented here.

In consequence, if indeed the determination of the 'freeze-out' point by use of net-charge cumulants is consistent with the 'freeze-out' point of the mean particle values, one can only conclude that either no correlations where present near the 'freeze-out' or they have been washed out by the rescattering. If the measurement shows practically independent particle production then no new information can be gained from the measurement of higher order cumulants as compared to the mean particle multiplicities.

\section{Summary}

We have presented a summary of the dynamics in the hadronic re-scattering phase of the UrQMD transport model. The hadronic re-scattering has a substantial ( $30 \%$ ) impact on most hadronic observables. In particular the calculated resonance yields which reproduce well the experimental data verify the existence of the hadronic phase experimentally. Furthermore the spectra and yields of stable hadrons are changed significantly, e.g. the proton number decreases by about $20 \%$ due to the annihilation of nucleons and excited baryonic states with their anti-particles. We also observe significant $(\sim 20 \%)$ modifications for the elliptic flow $v_{2}$ during the hadronic phase. We found that the hadronic rescattering phase leads to a substantial decorrelation of the conserved charge distributions. This effect is present for all investigated conserved charges and is mostly energy independent. This means that the final distribution function, the only one which can be observed, is to a large extent uncorrelated to that of the newly born hadrons which may carry information on the quark phase of QCD. These observations show that the experimental results cannot assert, or be directly compared to, the properties of the system at a conjectured hadronization or chemical freeze-out point.

\section{Acknowledgments}

V.V. appreciates the support from HGS-HIRe for FAIR. H.St. appreciates the support from J.M. Eisenberg Laureatus chair. The computational resources were provided by the LOEWE Frankfurt Center for Scientific Computing (LOEWE-CSC), and by the Kronos computing cluster at GSI.

\section{References}

[1] M. Gyulassy and L. McLerran, Nucl. Phys. A 750, 30 (2005) 
[2] J. Adams et al. [STAR Collaboration], Nucl. Phys. A 757, 102 (2005)

[3] B. B. Back et al., Nucl. Phys. A 757, 28 (2005)

[4] I. Arsene et al. [BRAHMS Collaboration], Nucl. Phys. A 757, 1 (2005)

[5] K. Adcox et al. [PHENIX Collaboration], Nucl. Phys. A 757, 184 (2005)

[6] C. Gale, S. Jeon and B. Schenke, arXiv:1301.5893 [nucl-th].

[7] K. Werner, I. Karpenko, T. Pierog, M. Bleicher and K. Mikhailov, Phys. Rev. C 82, 044904 (2010)

[8] C. Shen, Z. Qiu, H. Song, J. Bernhard, S. Bass and U. Heinz, Comput. Phys. Commun. 199, 61 (2016)

[9] H. Petersen, J. Steinheimer, G. Burau, M. Bleicher and H. Stocker, Phys. Rev. C 78, 044901 (2008)

[10] S. A. Bass and A. Dumitru, Phys. Rev. C 61, 064909 (2000)

[11] D. Teaney, J. Lauret and E. V. Shuryak, Phys. Rev. Lett. 86, 4783 (2001)

[12] T. Hirano, U. W. Heinz, D. Kharzeev, R. Lacey and Y. Nara, Phys. Lett. B 636, 299 (2006)

[13] C. Nonaka, AIP Conf. Proc. 1235, 165 (2010).

[14] T. Hirano, P. Huovinen and Y. Nara, Phys. Rev. C 83, 021902 (2011)

[15] S. Ryu, S. Jeon, C. Gale, B. Schenke and C. Young, arXiv: 1210.4588 [hep-ph].

[16] A. G. Knospe, C. Markert, K. Werner, J. Steinheimer and M. Bleicher, Phys. Rev. C 93, no. 1, 014911 (2016)

[17] J. Steinheimer, J. Aichelin and M. Bleicher, Phys. Rev. Lett. 110, 042501 (2013)

[18] J. Steinheimer, J. Aichelin and M. Bleicher, EPJ Web Conf. 36, 00002 (2012).

[19] A. Ilner, D. Cabrera, C. Markert and E. Bratkovskaya, Phys. Rev. C 95, no. 1, 014903 (2017)
[20] J. Steinheimer, J. Aichelin, M. Bleicher and H. Stöcker, Phys. Rev. C 95, no. 6, 064902 (2017)

[21] J. Steinheimer, V. Vovchenko, J. Aichelin, M. Bleicher and H. Stöcker, arXiv:1608.03737 [nucl-th].

[22] P. Huovinen and H. Petersen, arXiv:1206.3371 [nuclth].

[23] C. Patrignani et al. [Particle Data Group], Chin. Phys. C 40, no. 10, 100001 (2016).

[24] S. A. Bass et al., Prog. Part. Nucl. Phys. 41, 255 (1998) [Prog. Part. Nucl. Phys. 41, 225 (1998)]

[25] M. Bleicher et al., J. Phys. G25, 1859 (1999)

[26] M. Bleicher and J. Aichelin, Phys. Lett. B 530, 81 (2002)

[27] J. Adams et al. [STAR Collaboration], Phys. Rev. C 71, 064902 (2005)

[28] B. I. Abelev et al. [STAR Collaboration], Phys. Rev. C 78, 044906 (2008)

[29] B. I. Abelev et al. [STAR Collaboration], Phys. Rev. Lett. 97, 132301 (2006)

[30] R. Rapp and E. V. Shuryak, Phys. Rev. Lett. 86, 2980 (2001)

[31] C. Greiner and S. Leupold, J. Phys. G27, L95 (2001)

[32] W. Cassing, Nucl. Phys. A 700, 618 (2002)

[33] L. M. Satarov, I. N. Mishustin and W. Greiner, Phys. Rev. C 88, 024908 (2013)

[34] P. Braun-Munzinger, J. Stachel and C. Wetterich, Phys. Lett. B 596, 61 (2004)

[35] Y. Pan and S. Pratt, arXiv:1210.1577 [nucl-th].

[36] F. Karsch and K. Redlich, Phys. Lett. B 695, 136 (2011)

[37] P. Braun-Munzinger, A. Kalweit, K. Redlich and J. Stachel, Phys. Lett. B 747, 292 (2015)

[38] P. Alba, R. Bellwied, M. Bluhm, V. Mantovani Sarti, M. Nahrgang and C. Ratti, Phys. Rev. C 92, no. 6, 064910 (2015)

[39] A. Bzdak, V. Koch and N. Strodthoff, Phys. Rev. C 95, no. 5, 054906 (2017) 